\title{
Evidências sobre relaxantes musculares de uso ambulatorial: Uma revisão da literatura
}

\author{
Evidences on muscular relaxants for outpatient use: A review of the literature
}

Evidencias sobre relajantes musculares para el uso ambulatorio: Una revisión de la literatura

\author{
Lívia Helena Freitas da Silva Cascaes, Jardel Corrêa de Oliveira
}

\section{Resumo}

Objetivo: Avaliar as evidências sobre eficácia e efeitos adversos dos relaxantes musculares de uso oral disponíveis no Brasil para espasticidade, condições musculoesqueléticas, fibromialgia e cefaleia tensional. Métodos: Realizou-se uma revisão da literatura a partir de revisões sistemáticas publicadas no Medline, BVS, biblioteca Cochrane e National Institute for Health and Care Excellence (NICE) até dezembro de 2016, que avaliaram os fármacos considerados relaxantes musculares pela Anatomical Therapeutic Chemical (ATC) e disponíveis no Brasil na forma oral: ciclobenzaprina, tizanidina, carisoprodol, orfenadrina e baclofeno. Resultados: Foram identificados 20 estudos, sendo 17 revisões sistemáticas e três meta-análises. As evidências de eficácia dos relaxantes musculares consistem principalmente em estudos com concepção metodológica ruim. Estudos de comparação não mostraram que um relaxante muscular esquelético seja superior a outro. Ciclobenzaprina demonstrou eficácia em condições musculoesqueléticas, como dor miofascial mandibular, fibromialgia e dor lombar. Na fibromialgia, demonstrou benefício na melhora geral e no sono. No manejo da dor lombar, a ciclobenzaprina mostrou efeito modesto, mais presente nos quatro primeiros dias. Carisoprodol na dor lombar baixa não parece ter diferença de ciclobenzaprina, mas esse medicamento pode causar dependência. Baclofeno e tizanidina parecem ser eficazes em comparação com placebo e equivalentes em doentes com espasticidade. Conclusões: Os relaxantes musculares em geral, comparados a placebo ou entre si, apresentaram poucas evidências com estatística significante. Portanto, a seleção do medicamento deve ser baseada no perfil de efeitos adversos, preferência do paciente, potencial de abuso, potencial de interação com outros medicamentos, custo e outras características dos fármacos.

\section{Abstract}

Objective: To evaluate the evidence on the efficacy and adverse effects of oral muscle relaxants available in Brazil for spasticity, musculoskeletal conditions, fibromyalgia and tension headache. Methods: A literature review assessment was carried out based on systematic reviews published in the Medline, Virtual Health Library (VHL), Cochrane Library and National Institute for Health and Care Excellence (NICE) up to December 2016, that evaluated the drugs considered to be muscle relaxants by Anatomical Therapeutic Chemical (ATC) and available in Brazil in oral form: cyclobenzaprine, tizanidine, carisoprodol, orphenadrine and baclofen. Results: Twenty studies were identified, 17 of which were systematic reviews and three meta-analyzes. Evidence on the efficacy of muscle relaxants consists mainly of studies with poor methodological design. Comparison studies have not shown that one skeletal muscle relaxant is superior to another. Cyclobenzaprine demonstrated efficacy in musculoskeletal conditions, such as myofascial mandibular pain, fibromyalgia and low back pain. In fibromyalgia, it has shown benefit in overall improvement and sleep. In the management of low back pain, cyclobenzaprine showed modest effect, more present in the first four days. Carisoprodol in low back pain does not appear to have difference for cyclobenzaprine but this drug may cause dependence. Baclofen and tizanidine appear to be efficacious compared to placebo and equivalent in patients with spasticity. Conclusions: Muscle relaxants in general compared to placebo or to each other showed little evidence with significant statistics. Therefore the drug selection should be based on the profile of adverse effects, patient preference, potential of abuse, potential of interaction with other drugs, cost and other characteristics of the drugs.

Como citar: Cascaes LHFS, Oliveira JC. Evidências sobre relaxantes musculares de uso ambulatorial: Uma revisão da literatura. Rev Bras Med Fam Comunidade. 2017;12(39):1-14. http://dx.doi.org/10.5712/rbmfc12(39)1500
Palavras-chave:

Fármacos Neuromusculares

Avaliação de Resultado de

Intervenções Terapêuticas

Assistência Ambulatorial

Medicamentos para a

Atenção Primária

Revisão

Keywords:

Neuromuscular Agents

Evaluation of Results of

Therapeutic Interventions Ambulatory Care

Drugs for Primary Health Care Review

Fonte de financiamento: declaram não haver. Parecer CEP: não se aplica. Conflito de interesses: declaram não haver. Procedência e revisão por pares: revisado por pares. Recebido em: 20/03/2017. Aprovado em: 27/11/2017. 


\section{Resumen}

Objetivo: Evaluar las evidencias sobre eficacia y efectos adversos de los relajantes musculares de uso oral disponibles en Brasil para espasticidad, condiciones musculoesqueléticas, fibromialgia y cefalea tensional. Métodos: Se realizó una revisión de literatura a través de las revisiones sistemáticas publicadas en Medline, BVS, Cochrane Library y en el National Institute for Health and Care Excellence (NICE), hasta diciembre de 2016, que evaluó los fármacos considerados relajantes musculares por la Anatomical Therapeutic Chemical (ATC) y disponible en Brasil en forma oral: ciclobenzaprina, tizanidina, carisoprodol, orfenadrina y baclofeno. Resultados: Se identificaron 20 estudios, de los cuales 17 son revisiones sistemáticas y tres meta-análisis. La evidência de la eficácia de los relajantes musculares se compone principalmente de estudios de un mala concepción metodológica. Los estudios de comparación no mostraron que un relajante muscular esquelético es superior a otro. La ciclobenzaprina ha demostrado eficacia en los trastornos musculoesqueléticos, como dolor miofascial mandibular, fibromialgia y dolor lumbar. En la fibromialgia, demostró un beneficio en la mejora general y el sueño. En el tratamiento del dolor lumbar, ciclobenzaprina mostró efecto modesto, más presente en los primeros cuatro días. El carisoprodol, en el dolor lumbar, no parece tener diferencia de la ciclobenzaprina, pero este fármaco puede causar dependencia. El baclofeno y la tizanidina parecen ser eficaces en comparación con placebo y equivalentes en pacientes con espasticidad. Conclusiones: Los relajantes musculares en general en comparación con placebo o entre sí mostraron poca evidencia con estadísticas significativas. Por lo tanto, la selección de los medicamentos debe basarse en el perfil de efectos adversos, la preferencia del paciente, el potencial de abuso, el potencial de la interacción con otros medicamentos, costo y otras características de los fármacos.

\section{Palabras clave:}

Fármacos Neuromusculares Evaluación de Resultados de Intervenciones Terapéuticas Atención Ambulatoria Medicamentos para Atención Básica Revisión

\section{Introdução}

Os relaxantes musculares formam um grupo heterogêneo de medicamentos com um amplo espectro de indicações terapêuticas. Como descrito na literatura, são utilizados para tratar condições musculoesqueléticas periféricas, dores neuropáticas (por exemplo, a neuralgia trigeminal), espasticidade, fibromialgia, cefaleia tensional, discinesias tardias, ataxia etc. ${ }^{1,2}$ Muitas vezes, esse grupo de medicamentos é utilizado como adjuvante na ampliação do arsenal terapêutico das dores crônicas com intenção, muitas vezes, de superar uma monoterapia centrada apenas em analgésicos ou anti-inflamatórios. ${ }^{2}$

Segundo o guia da Associação da Indústria Farmacêutica de Pesquisa, no ano de 2016, entre os cinco medicamentos mais vendidos nas farmácias brasileiras estavam em primeiro e quinto lugar da lista uma associação de relaxante muscular com analgésico. ${ }^{3}$ Dessa forma, o tema possui grande relevância, pois os miorrelaxantes ocupam espaço importante no enfrentamento da dor e são frequentemente utilizados em ambulatório. Atualmente, a maioria dos relaxantes musculares não está disponível no SUS, apenas o diazepam, que possui risco de adicção. ${ }^{4}$ Portanto, é importante que seja avaliada a inclusão de medicamentos dessa classe na Relação Nacional de Medicamentos Essenciais (RENAME).

As dores de origem musculoesquelética periférica possuem grande prevalência entre jovens e constumam aumentar com a idade. ${ }^{5}$ Quanto à espasticidade, a incidência e prevalência apresentam taxas variadas e estão intimamente relacionadas com as doenças correspondentes. Não há dados epidemiológicos oficiais no Brasil, porém, de acordo com a literatura internacional, a prevalência da espasticidade em pacientes incapacitados é muito alta. ${ }^{6}$

Dessa forma, existem dois tipos de condições principais em que os relaxantes musculares são comumente utilizados: a espasticidade causada por síndromes do neurônio motor superior e a dor muscular ou espasmos derivados de condições musculoesqueléticas periféricas. Embora sejam do mesmo grupo, a Food and Drug Administration (FDA) aprovou poucos medicamentos dessa classe para o tratamento da espasticidade (como o dantrolene e o baclofeno), sendo o restante aprovado para o tratamento de condições musculoesqueléticas. Em geral, os fármacos antiespásticos ou antiespasmódicos não são 
permutáveis e não devem ser substituídos uns pelos outros. No entanto, diazepam e tizanidina possuem indicação aprovada pela FDA para ambas as condições. Entender essas diferenças é importante, pois ajuda na escolha do medicamento apropriado. ${ }^{7}$

Assim, o objetivo desta revisão foi avaliar as evidências científicas disponíveis sobre a eficácia e efeitos adversos dos relaxantes musculares para espasticidade, condições musculoesqueléticas, fibromialgia e cefaleia tensional disponíveis no Brasil na forma oral que pudessem ser utilizados na Atenção Primária à Saúde (APS) e futuramente incorporados nas listas de medicamentos essenciais do SUS.

\section{Métodos}

Realizou-se uma revisão da literatura sobre a eficácia dos fármacos considerados relaxantes musculares pela Anatomical Therapeutic Chemical (ATC) $)^{8}$ e com forma farmacêutica oral registrada na Agência Nacional de Vigilância Sanitária do Brasil (ANVISA), ${ }^{9}$ conforme diretrizes metodológicas para elaboração de pareceres técnico-científicos do Ministério da Saúde. ${ }^{10}$ Dessa forma, foram selecionados como objetos de estudo os seguintes medicamentos: ciclobenzaprina, tizanidina, carisoprodol, orfenadrina e baclofeno. Diazepam foi excluído das intervenções avaliadas porque além de já fazer parte da RENAME, não é um fármaco citado pela ATC como relaxante muscular e possui elevado risco de abuso pelos usuários.

De acordo com o modelo PICO,${ }^{10}$ buscou-se por estudos que avaliaram a eficácia na melhora da espasticidade, da dor articular e musculoesquelética, da cefaleia tensional e fibromialgia dos relaxantes musculares acima comparados entre si, com placebo, com analgésicos ou anti-inflamatórios, desde que os relaxantes musculares fossem a única intervenção diferente entre os dois grupos de comparação.

As evidências disponíveis sobre os fármacos pesquisados foram avaliadas considerando as suas indicações registradas pela ANVISA: fibromialgia, dor articular e musculoesquelética (dor lombar, torcicolo, contratura muscular, dor miofascial, gota, artrite reumatoide e outras artrites), cefaleia tensional, espasticidade (por trauma medular ou cerebral, por doenças neurológicas degenerativas ou paralisia cerebral).

Assim, as evidências foram analisadas em relação a dois tipos de condições: a espasticidade causada por síndromes do neurônio motor superior e a dor muscular ou espasmos derivados de condições musculoesqueléticas periféricas. Como desfechos foram considerados: melhora da dor, da espasticidade, melhora funcional e efeitos adversos. Os descritores para cada um dos relaxantes musculares pesquisados e sinônimos foram obtidos no Mesh e Decs.

Realizou-se uma busca por meta-análises (MA) e revisões sistemáticas (RS) no Medline via PubMed, na Biblioteca Virtual em Saúde (BVS), na Biblioteca Cochrane e National Institute for Health and Care Excellence (NICE). Não houve restrição de idioma, sendo os limites: estudos publicados até dezembro de 2016, humanos e tipo de estudo. A estratégia de busca utilizada foi: "carisoprodol or isopropylmeprobamate or orphenadrine or mefenamine or baclofen or "Chlorophenyl GABA" or tizanidine or cyclobenzaprine".

Os estudos identificados foram agrupados no software de manejo de referências EndNote versão X5, sendo retiradas as duplicatas. Foram excluídos estudos com população composta por animais, indicações terapêuticas não reconhecidas pela ANVISA, intervenções diferentes daquelas consideradas na estratégia de busca, comparadores distintos do PICO e ainda estudos com outro tipo de desenho que não MA ou RS. 
Os estudos foram identificados de acordo com título e resumo quanto ao preenchimento dos critérios de seleção. Quando o título ou o resumo não permitiu a exclusão e o estudo estava disponível na íntegra, a avaliação foi feita com base na metodologia do artigo. Quando o texto completo não estava disponível, tentou-se obter o estudo pelo portal CAPES na Universidade Federal de Santa Catarina ou da McMaster University do Canadá, sendo então excluídos os estudos que não foram obtidos. A Tabela 1 demonstra o processo de seleção.

Tabela 1. Seleção das revisões sistemáticas e meta-análises.

\begin{tabular}{|ccccccc|}
\hline & \multicolumn{4}{c|}{ Estudos que não preencheram critérios de seleção após análise do título e/ou do resumo } \\
ou da metodologia
\end{tabular}

Quanto aos seis estudos cujos resumos e/ou o texto completo não estavam disponíveis, quatro deles abordavam o uso de tizanidine e outras drogas ${ }^{11-14}$ (três deles publicados na década de 90 abordavam espasticidade e um de 2004 abordava dor miofascial dos músculos mastigatórios). Outro estudo, publicado em 2003, abordou o uso de carisoprodol e seu potencial de abuso. ${ }^{15} \mathrm{Em}$ seu resumo relata que a revisão encontrou poucas evidências para apoiar o uso do carisoprodol no controle da dor. O sexto estudo excluído abordava ciclobenzaprina e outras drogas para tratamento de fibromialgia ${ }^{16}$ e foi publicado em 1989.

\section{Resultados e Discussão}

Foram identificados 20 estudos para incluir nesta revisão, sendo 17 RS e três MA. As características dos estudos incluídos estão descritas no Quadro 1.

\section{Fibromialgia}

Foram encontradas duas MA que avaliaram a eficácia de ciclobenzaprina no tratamento da fibromialgia. ${ }^{17,18}$ Embora, uma delas tenha realizado busca mais recente, ${ }^{18}$ incluiu um número menor de estudos, que já estavam descritos na MA mais antiga.

AMA $^{17}$ com maior número de estudos $(n=312)$ reuniu cinco ensaios clínicos controlados randomizados (ECR) e avaliou ciclobenzaprina versus placebo. Três desses estudos foram reunidos e demonstraram diferença estatisticamente significativa na autopercepção de melhora geral dos pacientes (OR 3,0; IC $95 \% 1,6-5,6)$. Estes resultados foram homogêneos ( $p=0,53$, mas com evidência de viés de publicação, $\mathrm{p}=0,05)$. A diferença de risco foi de 0,21 (IC 95\% 0,09-0,34) e o NNT de 4,8 (IC 95\% 3,0-11), porém não foram relatados os números absolutos para confirmar essa informação.

Em relação aos dados contínuos extraídos dos cinco estudos dessa $\mathrm{MA},{ }^{17}$ relatou-se uma melhora estatisticamente significativa no sono para todos os pontos de tempo (4, 8 e 12 semanas) com diferença média padronizada (DMP) variando de 0,34 a 0,50. A dor teve melhora no grupo de ciclobenzaprina apenas na quarta semana com $\mathrm{DMP}=0,35$, sendo que autores consideraram haver risco de viés de publicação. 
Quadro 1. Estudos incluídos

\begin{tabular}{|c|c|c|}
\hline População & Revisões sistemáticas & Intervenção \\
\hline \multirow{2}{*}{ Fibromialgia } & $\begin{array}{l}\text { Tofferi JK, Jackson JL, O'Malley PG. (2004) } \\
\text { Meta-análise }\end{array}$ & Ciclobenzaprina vs. placebo \\
\hline & $\begin{array}{l}\text { Roskell NS, Beard SM, Zhao Y, Trong KL. } \\
(2012)^{18} \text { - Meta-análise }\end{array}$ & Ciclobenzaprina vs. placebo \\
\hline Artrite Reumatoide & Richards BL, Whittle SL, Buchbinder R. (2012) ${ }^{19}$ & $\begin{array}{l}\text { Orfenadrina, carisoprodol, ciclobenzaprina, } \\
\text { baclofeno e tizanidina - nenhum ECR encontrado }\end{array}$ \\
\hline Esclerose Lateral Amiotrófica & Baldinger R, Katzberg, HD, Weber, M. (2012) ${ }^{20}$ & Baclofeno vs. placebo \\
\hline Dor Miofascial & $\begin{array}{l}\text { Leite FM, Atallah AN, El Dib R, Grossmann E, } \\
\text { Januzzi E, Andriolo RB, et al. }(2009)^{22}\end{array}$ & Ciclobenzaprina vs. placebo \\
\hline $\begin{array}{l}\text { Desordens Mecânicas do } \\
\text { Pescoço }\end{array}$ & $\begin{array}{l}\text { Peloso P, Gross A, Haines T, Trinh K, Goldsmith } \\
\mathrm{CH} \text {, Aker P. }(2005)^{21}\end{array}$ & Ciclobenzaprina vs. placebo \\
\hline \multirow{3}{*}{ Dor Lombar } & $\begin{array}{l}\text { Vroomen PCAJ, De Krom MCTFM, Slofstra PD, } \\
\text { Knottnerus JA (2002) }{ }^{24}\end{array}$ & Tizanidina vs. placebo \\
\hline & $\begin{array}{l}\text { Luijsterburg PAJ, Verhagen AP, Ostelo RWJG, Van } \\
\text { Os TAG, Peul WC, Koes BW. }(2008)^{25}\end{array}$ & Tizanidina vs. placebo \\
\hline & $\begin{array}{l}\text { Browning R, Jackson JL, O'Malley PG. (2001) } \\
\text { Meta-análise }\end{array}$ & Ciclobenzaprina vs. placebo \\
\hline $\begin{array}{l}\text { Espasticidade e Desordens } \\
\text { Musculoesqueléticas }\end{array}$ & Chou R, Peterson K, Helfand M. (2004) ${ }^{23}$ & $\begin{array}{l}\text { Orfenadrina, carisoprodol, ciclobenzaprina, } \\
\text { tizanidina ou baclofeno vs. placebo, } \\
\text { ciclobenzaprina vs. carisoprodol e tizanidina vs. } \\
\text { baclofeno }\end{array}$ \\
\hline \multirow{3}{*}{ Esclerose Múltipla } & Shakespeare DT, Boggild M, Young C. (2003) & $\begin{array}{l}\text { Baclofeno ou tizanidina vs. placebo e tizanidina } \\
\text { vs. baclofeno }\end{array}$ \\
\hline & Beard S, Hunn A, Wight J. (2003) ${ }^{29}$ & $\begin{array}{l}\text { Baclofeno ou tizanidina vs. placebo e tizanidina } \\
\text { vs. baclofeno }\end{array}$ \\
\hline & Paisley S, Beard S, Hunn A, Wight J. (2002) ${ }^{28}$ & $\begin{array}{l}\text { Baclofeno ou tizanidina vs. placebo e tizanidina } \\
\text { vs. baclofeno }\end{array}$ \\
\hline \multirow{2}{*}{ Paralisia Cerebral } & $\begin{array}{l}\text { Delgado MR, Hirtz D, Aisen M, Ashwal S, Fehlings } \\
\text { DL, McLaughlin J, et al. }(2010)^{30}\end{array}$ & Baclofeno ou tizanidina vs. placebo \\
\hline & $\begin{array}{l}\text { Navarrete-Opazo AA, Gonzalez W, Nahuelhual P. } \\
(2016)^{31}\end{array}$ & Baclofeno vs. placebo \\
\hline Lesão Medular & $\begin{array}{l}\text { Taricco M, Pagliacci MC, Telaro E, Adone RT. } \\
(2006)^{33}\end{array}$ & Baclofeno ou tizanidina vs. placebo \\
\hline \multirow{3}{*}{ Lesão Cerebral } & Olvey EL, Armstrong EP, Grizzle AJ. (2010) ${ }^{36}$ & Tizanidina vs. placebo \\
\hline & $\begin{array}{l}\text { Lindsay C, Kouzouna A, Simcox C, Pandyan AD. } \\
(2016)^{35}\end{array}$ & Tizanidina vs. placebo ou baclofeno \\
\hline & $\begin{array}{l}\text { Marshall S, Teasell R, Bayona N, Lippert C, } \\
\text { Chundamala J, Villamere J, et al. (2007) }{ }^{34}\end{array}$ & Baclofeno ou tizanidina vs. placebo \\
\hline $\begin{array}{l}\text { Lesão Cerebral ou Medular e } \\
\text { Paralisia Cerebral }\end{array}$ & Montané E, Vallano A, Laporte JR. (2004) ${ }^{32}$ & $\begin{array}{l}\text { Baclofeno ou tizanidina vs. placebo e baclofeno } \\
\text { vs. Tizanidina }\end{array}$ \\
\hline
\end{tabular}

Um efeito de 0,2 foi considerado pequeno e de 0,5 moderado. Nenhuma melhora na fadiga ou nos pontos de sensibilidade foi observada em qualquer momento. Essas análises foram homogêneas para fadiga, sono, dor e pontos sensíveis em todos os períodos de tempo.

Essa $\mathrm{MA}^{17}$ apresentou muitas limitações. Entre os cinco estudos incluídos, três não indicaram método de randomização, dois não continham descrição clara dos critérios de exclusão e dois não realizaram análise por intenção de tratar. Um deles continha descrição inadequada das perdas e os outros quatro tiveram 
grandes perdas de seguimento. Em média, apenas $71 \%$ dos pacientes tratados com ciclobenzaprina e $57 \%$ com placebo completaram os estudos. Em geral, a qualidade dos estudos incluídos foi considerada pelos autores como moderada pela escala Jadad. No grupo ciclobenzaprina, $85 \%$ dos pacientes tiveram reações adversas, que não foram descritas.

\section{Artrite Reumatoide}

Foi incluída apenas uma $\mathrm{RS}^{19}$ que avaliou o uso de relaxantes musculares no manejo da dor em adultos com diagnóstico de artrite reumatoide. Porém, até 2010, quando se encerrou busca ampla em várias bases de dados e com especialistas na área, não haviam sido encontrados estudos que envolvessem a artrite reumatoide e as intervenções de interesse dessa revisão.

\section{Esclerose Lateral Amiotrófica}

Foi encontrada uma $\mathrm{RS}^{20}$ da Cochrane que avaliou o tratamento de câimbras em indivíduos com esclerose lateral amiotrófica. Nessa RS, foi encontrado um ECR $(n=20)$ duplo-cego paralelo, com seguimento de sete semanas, que comparou baclofeno 15 a 80 mg/dia versus placebo e não demonstrou benefício. Esse ECR apresentou informações insuficientes para avaliar se a randomização, o sigilo de alocação e o cegamento foram adequados.

\section{Desordens mecânicas do pescoço}

Foi encontrada uma $\mathrm{RS}^{21}$ da Cochrane que avaliou o tratamento de adultos com desordens mecânicas no pescoço associada ou não com cefaleia ou síndrome radicular. Nessa RS foi incluído apenas um ECR $(n=22)$ duplo-cego paralelo, que avaliou ciclobenzaprina 10mg (três vezes por dia) versus placebo com tempo de tratamento de duas semanas, que não demonstrou benefício na melhora do espasmo muscular por avaliação de escala de cinco pontos. Esse estudo não apresentou informações suficientes para avaliar o número de perdas e se a randomização e o sigilo de alocação foram adequados. Quanto aos efeitos adversos, no grupo tratado com ciclobenzaprina foi relatada sensação de boca seca e sonolência sem informações quantitativas a respeito.

\section{Dor Miofascial}

Foi encontrada uma $\mathrm{RS}^{22}$ da Cochrane que avaliou o tratamento de adultos com diagnóstico clínico de dor miofascial. Nessa $\mathrm{RS}^{22}$ foi descrito um ECR $(n=41)$ duplo-cego paralelo, com seguimento de três semanas, que avaliou ciclobenzaprina $10 \mathrm{mg}$ (uma vez ao dia) versus placebo no tratamento da dor miofascial mandibular. Apesar do pequeno número de participantes, o estudo foi de boa qualidade (sem diferenças significativas de base entre os grupos de comparação, sem perdas substanciais de seguimento e com randomização, cegamento e sigilo de alocação adequados). Esse ECR demonstrou diferença de ciclobenzaprina sobre placebo na melhora na intensidade da dor mandibular, diferença média (DM) -0,25 (IC $95 \%,-0,41$ a -0,09; $p=0,002)$. Quanto à avaliação da qualidade do sono e ocorrência de efeitos adversos, não houve diferença significativa. 


\section{Dor Lombar}

Ao total foram encontradas três $\mathrm{RS}^{23-25}$ e uma $\mathrm{MA}^{26}$ que envolveram a avaliação da dor lombar com intervenções de interesse dessa revisão.

$\mathrm{AMA}^{26}$ avaliou ciclobenzaprina versos placebo na melhora da dor lombar. Foram incluídos 14 ECR de moderada qualidade segundo o autor, pela escala de Jadad. Desses, 10 ECR foram agrupados e relataram benefício com ciclobenzaprina para melhora global da dor lombar (até o 10 dia de tratamento) com OR de 4,7 (IC 95\% 2,7-8,1), diferença de risco de 0,37 (IC 95\% 0,24-0,50) e NNT de 2,7 (IC 95\% 2,0-4,2). A dose média diária de ciclobenzarina foi de $30 \mathrm{mg}$ e em geral dividia em três vezes ao dia. Há evidência de heterogeneidade entre as amostras dos estudos ( $p=0,002)$, porém não houve viés de publicação $(p=0,37)$.

Além disso, a partir desses 10 ECR foram avaliadas 5 variáveis contínuas em 3 pontos diferentes (de 1 a 4 dias, de 5 a 9 dias e mais que 9 dias) para mensurar a melhora dos pacientes quanto a dor local, espasmo muscular, rigidez à palpação, amplitude de movimento e atividades diárias. Nos três pontos avaliados os pacientes tiveram melhora significativa para as cinco variáveis.

Para quatro dessas variáveis (espasmo muscular, rigidez a palpação, amplitude de movimento e atividades diárias), houve uma grande diferença entre ciclobenzaprina e placebo nos primeiros três dias em relação a qualquer outro ponto de tempo. Nos primeiros dias de tratamento essa melhora foi quantificada pelo autor como moderada $(0,52)$ e após foi reduzida $(0,44)$. Em contexto, um efeito de 0,2 foi considerado pequeno, 0,5, moderado e 0,8, grande.

Dessa maneira, embora não tenha sido relatada diferença significativa entre os três pontos de medidas das variáveis, houve evidência estatística da tendência de perda de eficácia através do tempo de ciclobenzaprina $(p=0,04)$. Não houve evidência estatística de heterogeneidade para nenhum dos pontos avaliados embora tenha ocorrido viés de publicação nos primeiros dias de avaliação (até o quarto dia) para as variáveis: dor local, espasmo muscular, rigidez a palpação e amplitude de movimento. ${ }^{26}$

Essa $M^{26}$ possui sérias limitações, já que não especificou o número de participantes e não forneceu números absolutos exemplificando os cálculos obtidos. Os pacientes tratados com ciclobenzaprina foram significativamente mais propensos a relatarem efeitos adversos. Ao total, $53 \%$ dos pacientes tratados com ciclobenzaprina tiveram pelo menos uma reação adversa contra $28 \%$ com placebo. As mais relatadas foram sonolência $(p<0,001)$, boca seca $(p=0,02)$ e tontura $(p=0,04)$.

Em relação às três $R S$ encontradas, uma delas ${ }^{23}$ avaliou diversas intervenções de interesse dessa revisão na melhora da dor lombar. Excluídos os ECR incluídos na MA ${ }^{26}$ já mencionada, nessa $\mathrm{RS}^{23}$ foram citados mais oito ECR que avaliaram orfenadrina, carisoprodol, baclofeno e ciclobenzaprina descritos no Quadro 2.

As outras duas $\mathrm{RS}^{24,25}$ avaliaram tizanidina na dor lombar aguda. Cada uma incluiu um ECR diferente que avaliou tizanidina $12 \mathrm{mg} /$ dia versos placebo por sete dias e ambos não evidenciaram diferença significativa.

\section{Espasticidade na Esclerose Múltipla}

Foram encontradas quatro $\mathrm{RS}^{23,27-29}$ que avaliaram o tratamento da espasticidade na esclerose múltipla (EM). Todas avaliaram tizanidina e baclofeno versus placebo e tizanidina versus baclofeno. 
Quadro 2. Descrição ECR.

\begin{tabular}{|c|c|c|c|}
\hline Autor & Intervenção & $\mathbf{n}$ & Desfecho \\
\hline Dapas (1985) & baclofeno vs. placebo & $(n=200)$ & $\begin{array}{l}\text { Baclofeno foi superior para dor lombar, pontos sensíveis, espasmo e } \\
\text { melhora funcional através de métodos não especificados }(p<0,05)\end{array}$ \\
\hline Rollings (1983) & ciclobenzaprina vs. carisoprodol & $(n=78)$ & $\begin{array}{l}\text { Sem diferença estatística entre as intervenções através de escala de } \\
\text { classificação verbal de } 5 \text { pontos e escala visual analógica. Sensação } \\
\text { de boca seca foi mais frequente com ciclobenzaprina (38\% vs. 10\%) e } \\
\text { tontura menos frequente ( } 8 \% \text { vs. } 26 \% \text { ). }\end{array}$ \\
\hline Baratta (1976) & carisoprodol vs. placebo & $(n=105)$ & $\begin{array}{l}\text { Não houve diferença significativa para dor através de avaliação por } \\
\text { escala de } 4 \text { pontos. Carisoprodol foi superior ao placebo na melhora do } \\
\text { sono e para medidas funcionais não especificadas. }\end{array}$ \\
\hline Cullen (1976) & carisoprodol vs. placebo & $(n=65)$ & $\begin{array}{l}\text { Carisoprodol foi superior para dor }(p<0,01) \text {, espasmo }(p<0,01) \\
\text { e limitação de movimento }(p<0,01) \text { através de métodos não } \\
\text { especificados. }\end{array}$ \\
\hline Hindle (1972) & carisoprodol vs. placebo & $(n=48)$ & $\begin{array}{l}\text { Carisoprodol foi superior para dor }(p<0,01) \text {, espasmo }(p<0,01) \text { e } \\
\text { avaliações funcionais }(p<0,01) \text { através de escala de } 4 \text { pontos e foi } \\
\text { superior na melhora da intensidade da dor usando escala visual } \\
\text { analógica de zero a cem }(p<0.01) \text {. }\end{array}$ \\
\hline Soyka (1979) & carisoprodol vs. placebo & $(n=414)$ & $\begin{array}{l}\text { Carisoprodol foi superior na melhora de espasmos musculares } \\
\text { ( } p=0,015) \text { e avaliação funcional }(p=0,04) \text { através de escala de } 5 \\
\text { pontos. Não foram encontradas diferenças significantes no sono por } \\
\text { escala de } 4 \text { pontos ou dor por escala de } 5 \text { pontos. }\end{array}$ \\
\hline Gold (1978) & orfenadrina vs. placebo & $(n=60)$ & $\begin{array}{l}\text { Artigo considerado de baixa qualidade. Orfenadrina foi superior na } \\
\text { melhora da intensidade e alívio da dor }(p<0,01) \text { através de métodos } \\
\text { não especificados. }\end{array}$ \\
\hline $\begin{array}{l}\text { Valtonen } \\
(1975)\end{array}$ & orfenadrina vs. placebo & $(n=200)$ & $\begin{array}{l}\text { Não houve diferença significativa através de escala de } 3 \text { pontos para } \\
\text { nenhuma medida. }\end{array}$ \\
\hline
\end{tabular}

Ao total, foi observado que apenas quatro ECR não foram incluídos na RS de Beard et al. ${ }^{29}$ Dois deles eram estudos não publicados que avaliaram baclofeno versus tizanidina, mas cujos dados não estavam disponíveis. Um ECR, com Jadad 2 pela $\mathrm{RS}^{28}$ que o incluiu, avaliou tizanidina versus placebo e blacofeno e não evidenciou diferença estatística entre as intervenções. Da mesma forma, o quarto ECR (Jadad 3) que avaliou tizanidina versus baclofeno também não obteve diferença estatística.

Dessa maneira, a RS de Beard et al. ${ }^{29}$ englobou 21 ECR incluídos nas outras RS. ${ }^{23,27,28}$ Desses ECR, nove avaliaram baclofeno 10 a $120 \mathrm{mg}$ versus placebo e quatro deles mostraram melhora estatisticamente significativa da espasticidade com baclofeno (Quadro 3). Os outros cinco ECR envolveram estudos de menor qualidade, com medidas não especificadas ou violação de protocolo. Embora pareça existir benefício de baclofeno, a evidência é limitada, pois a maioria dos estudos possui número de participantes pequeno e não houve menção de cálculos prévios de tamanho de amostra em nenhum deles.

Essa $\mathrm{RS}^{29}$ também incluiu 12 ECR que avaliaram tizanidina 2 a $36 \mathrm{mg}$ versus placebo ou baclofeno, que utilizaram uma ampla variedade de medidas de desfecho. Em cinco ECR duplo-cegos foi avaliado tizanidina versus placebo e em dois deles houve melhora estatisticamente significativa do resultado do teste do pêndulo, embora um deles, Nance et al. (1997), não tenha descrito adequadamente a melhora na escala Ashwort. Esses dois estudos estão descritos no Quadro 4. Os outros três ECR demonstraram melhora discreta da espasticidade em algumas avaliações e em outras não encontraram diferença estatística. Dessa forma, de modo geral, tizanidina parece ter benefício em relação ao placebo. 
Quadro 3. Características dos estudos envolvendo baclofeno versus placebo.

\begin{tabular}{|c|c|c|}
\hline Autor & $\stackrel{n}{\text { qualidade perda de seguimento }}$ & Desfecho \\
\hline $\begin{array}{l}\text { Feldman (1978) } \\
\text { (crossover) }\end{array}$ & $\begin{array}{c}n=33 \\
\text { Jadad } 3 \\
\text { PS: } 30 \%\end{array}$ & $\begin{array}{l}\text { Resistência ao movimento passivo: melhora em } 15 / 23 \text { do gupo baclofeno } \\
\text { vs. } 4 / 23 \text { no placebo }(p<0,05) \text {. Frequência dos espasmos: melhora em } 9 / 23 \\
\text { com baclofeno e } 1 / 23 \text { com placebo }(p<0.05) \text {. Clônus: melhora em } 12 / 23 \text { com } \\
\text { baclofeno e } 1 / 23 \text { com placebo }(p<0,001) \text {. Não houve diferença estatística } \\
\text { quanto à avaliação subjetiva de dor no membro, uso do membro espástico, } \\
\text { deambulação e índice de Barthel. Os efeitos adversos do baclofeno } \\
\text { incluiram sonolência, boca seca, parestesia e visão borrada sem descrição } \\
\text { especificada. }\end{array}$ \\
\hline $\begin{array}{l}\text { Sawa \& Paty (1979) } \\
\text { (crossover) }\end{array}$ & $\begin{array}{c}\mathrm{n}=21 \\
\text { Jadad } 3 \\
\text { PS: } 14 \%\end{array}$ & $\begin{array}{l}\text { Através de escala de cinco pontos para medir espasticidade: } 13 / 18 \text { pacientes } \\
\text { com baclofeno relataram melhora objetiva na espasticidade }(p<0,001) \text { sem } \\
\text { mudanças no grupo placebo. A incidência de efeitos adversos foi alta, } 71 \% \\
\text { dos pacientes do grupo baclofeno e } 19 \% \text { no grupo placebo. Os efeitos } \\
\text { adversos incluíram sedação, náuseas e vômitos. }\end{array}$ \\
\hline $\begin{array}{l}\text { Jerusalem (1968) } \\
\text { (paralelo) }\end{array}$ & $\begin{array}{c}\mathrm{n}=30 \\
\text { Jadad } 3 \\
\text { PS: zero }\end{array}$ & $\begin{array}{l}\text { Melhora em } 12 / 16 \text { pacientes do grupo baclofeno e } 5 / 14 \text { no placebo. Essa } \\
\text { diferença foi relatada como estatisticamente significativa, porém não } \\
\text { especificada. Os espasmos durante a noite melhoraram em } 8 / 9 \text { pacientes. } \\
\text { Dos } 25 \text { pacientes que fizeram uso de baclofeno (incluindo pacientes } \\
\text { transferidos do grupo placebo), } 7 \text { relataram sedação, } 5 \text { fraqueza, um náusea } \\
\text { e } 1 \text { boca seca. }\end{array}$ \\
\hline $\begin{array}{l}\text { Hudgson (1971) } \\
\text { (crossover) }\end{array}$ & $\begin{array}{c}n=23 \\
\text { Jadad } 4 \\
\text { PS: zero }\end{array}$ & $\begin{array}{l}\text { Houve melhora da espasticidade pela escala Ashwort no grupo baclofeno em } \\
16 \text { pacientes e no grupo placebo em apenas } 7 \text {. Isso significou uma mudança } \\
\text { na pontuação de } 1,44 \text { com baclofeno e } 0,54 \text { com placebo }(p<0,05) \text {. Em } \\
\text { análise subjetiva: } 13 \text { pacientes relataram melhora na rigidez com baclofeno, } \\
5 \text { com placebo e } 5 \text { não reportaram diferença. Efeitos adversos: náusea } \\
\text { moderada em seis pacientes com baclofeno e três com placebo. }\end{array}$ \\
\hline
\end{tabular}

* PS: perda de seguimento; ** n: número total de pacientes

Quadro 4. Características dos ECR de relevância tizanidina versus placebo.

\begin{tabular}{|c|c|c|}
\hline Autor & $\begin{array}{c}n \\
\text { qualidade perda de seguimento }\end{array}$ & Desfecho \\
\hline \multirow{2}{*}{ Nance et al. (1997) } & \multirow{2}{*}{$\begin{array}{l}\mathrm{n}=142 \\
\text { Jadad } 5 / 5 \\
\text { PS: } 1,4 \%\end{array}$} & $\begin{array}{l}\text { Índice de relaxamento baseado no teste do pêndulo: Melhora da resposta } \\
\text { relatada de acordo com a dose no grupo de tizanidina }\end{array}$ \\
\hline & & $\begin{array}{l}\text { Escala de força: sem alteração. Tônus muscular utilizando escala de Ashwort: } \\
\text { parece haver melhora moderada, porém sem outras especificações descritas. }\end{array}$ \\
\hline Emre et al. (1994) & $\begin{array}{l}\quad n=17 \\
\text { Jadad } 3 / 5 \\
\text { PS: } 0,5 \%\end{array}$ & $\begin{array}{l}\text { No grupo de tizanidina a pontuação na escala Ashwort diminuiu em média } \\
2 \text { pontos após } 1 \text { hora e } 1,6 \text { pontos após } 3 \text { horas }(p<0,001) \text {. Essa mudança } \\
\text { foi correlacionada com mudanças na concentração plasmática do fármaco. } \\
\text { Tizanidina produziu uma melhora significativa no teste do pêndulo }(p<0,001) \text {. }\end{array}$ \\
\hline
\end{tabular}

Os outros ECR dessa RS, ${ }^{29}$ sendo seis duplo-cegos e um parcialmente cego, avaliaram tizanidina versus baclofeno. Em todos esses estudos os resultados demonstraram semelhança dos efeitos na melhora da maioria das variáveis avaliadas. Tizanidina e baclofeno parecem ter eficácia semelhante, com pouca diferença em termos de frequência e gravidade dos efeitos adversos. Em geral, o efeito adverso mais frequente foi sonolência.

\section{Espasticidade na paralisia cerebral}

Foram encontradas duas $\mathrm{RS}^{30,31}$ que avaliaram tizanidina ou baclofeno versus placebo no tratamento da espasticidade em crianças e/ou adolescentes com paralisia cerebral (PC). Dois ECR se repetiram em 
ambas RS e oito estudos não. Uma terceira RS ${ }^{32}$ avaliou também espasticidade em doenças neurológicas não progressivas e incluiu um ECR já presente nas outras revisões.

Em ambas as $\mathrm{RS}^{30,31}$ não há dados suficientes para tirar conclusões sobre a eficácia do baclofeno oral no tratamento da PC espástica. Estudos mostram uma grande variabilidade de tamanho da amostra, classificação motora na PC, dose de baclofeno, duração total dos ensaios e medidas de resultado, bem como instrumentos de medidas utilizados. Embora a maioria dos estudos não apresentem diferenças significativas quanto a efeitos adversos entre baclofeno e placebo, a inconsistência dos resultados entre os estudos e a má qualidade global destes prejudicam essa análise.

Uma das $\mathrm{RS}^{30}$ avaliou tizanidina versus placebo para melhora da espasticidade em crianças e adolescentes. Apesar dessa RS ter apresentado que tizanidina é possivelmente eficaz com $p<0,0001$. Esse dado se baseou em apenas um ECR com 40 participantes. Portanto, não existe evidência suficiente para apoiar ou refutar o uso de tizanidina para melhorar a função motora nessa população.

Espasticidade na lesão medular

Foram encontradas três $\mathrm{RS}^{23,32,33}$ que incluíram cinco ECR envolvendo o tratamento da espasticidade após lesão medular. Duas $\mathrm{RS}^{32,33}$ incluíram ECR já presentes na revisão de Chou et al. ${ }^{23}$, com exceção de apenas um ECR incluído na RS de Taricco et al. ${ }^{33}$, que mostrou melhora com baclofeno comparado a placebo. Porém, envolveu apenas seis crianças, não descreveu a randomização, não mencionou critérios de exclusão e não relatou a análise estatística.

$\mathrm{ARS}^{23}$ avaliou a espasticidade de forma geral e englobou todos os outros quatro ECR que avaliaram especificamente a espasticidade na lesão medular. Em um desses estudos foi comparado tizanidina versus placebo e nos outros três baclofeno versus placebo. Dos três estudos envolvendo baclofeno, dois foram considerados pelo autor como de baixa qualidade e outro de moderada qualidade. Esse, por sua vez, demonstrou benefício do baclofeno, porém não relatou o valor de "p" e envolveu apenas seis participantes.

No outro ECR duplo-cego paralelo, considerado de moderada qualidade pelo autor, ${ }^{23}$ que comparou tizanidina versus placebo, avaliou-se a espasticidade após lesão medular em pacientes de 15 a 69 anos. Nesse estudo foi demonstrado beneficio de tizanidina comparada ao placebo em relação a pontuação na escala Ashworth $(-3,70$ SE 0,67$)(p<0,0001)$ e do teste do pêndulo $(p<0,004)$. Não houve diferença na frequência dos espasmos. No grupo de tizanidina, $81 \%$ dos pacientes relataram pelo menos um efeito adverso contra $53 \%$ no grupo placebo $(p=0,002)$. Os efeitos adversos mais comuns com tizanidina foram sonolência e boca seca $(p<0,001)$.

\section{Espasticidade na lesão cerebral}

Foram encontradas cinco $\mathrm{RS}^{23,32,34-36}$ que avaliaram o tratamento da espasticidade após lesão cerebral. Distribuídos entre essas RS, seis ECR diferentes foram descritos, dos quais dois ECR avaliaram a espasticidade após lesão cerebral por diversas causas (após trauma encefálico ou doença cérebro vascular, etc.) e não encontraram diferença significativa entre baclofeno versus tizanidina e entre baclofeno versus placebo. 
Os outros quatro ECR avaliaram a espasticidade especificamente após acidente vascular cerebral. Desses, um ECR que avaliou tizanidina versus placebo não encontrou diferença significativa entre os grupos e um outro ECR que avaliou baclofeno versus placebo especificamente em idosos foi interrompido devido à elevada ocorrência de efeitos adversos como sonolência em $76 \%$ do grupo de blacofeno. Dois ECR, com Jadad 4, tiveram achados de eficácia significativa. Um desses ECR $(n=17)$, duplo-cego crossover avaliou tizanidina 4-36mg versus placebo por 15 semanas e mostrou melhora com tizanidina na escala Ashworth $(p<0,0001)$, no tônus motor $(p=0,006)$ e na escala de espasmos de Penn $(p=0,046)$. O outro ECR $(n=20)$ duplo cego crossover avaliou baclofeno $30 \mathrm{mg} /$ dia versus placebo e encontrou benefício com baclofeno na escala de Ashwort $(p<0,001)$. Não foram detalhadas as limitações desses ECR.

\section{Contextualização ao cenário de prática da APS e Medicina de Familia no Brasil}

Apesar dos relaxantes musculares serem populares, a American Pain Society e a American College of Physicians recomendam utilizar paracetamol e anti-inflamatórios não esteroides como tratamento de primeira linha para dor lombar e reserva os relaxantes musculares como opção alternativa. ${ }^{37}$ Embora demonstrem benefício comparado a placebo para algumas condições clínicas avaliadas nessa revisão, não foram encontradas evidências comparando relaxantes musculares a outras classes de medicamentos ou como coadjuvantes. Portanto, é preciso individualizar seu uso na prática clínica conforme a resposta da pessoa, as opções de tratamento não farmacológico e farmacológico já disponíveis no sistema público de saúde, considerando as evidências existentes e o perfil de efeitos adversos dos relaxantes musculares.

Esse estudo não se propôs a avaliar o uso de outros recursos terapêuticos disponíveis no SUS, como diazepam, toxina botulínica, fisioterapia e práticas integrativas como acupuntura. Diazepam é um medicamento sujeito à prescrição controlada, que possui risco de abuso, não estando prontamente disponível na maioria dos ambulatórios de atenção primária para manejo de dor aguda. Toxina botulínica é utilizada em serviços de reabilitação, também não presente na APS. De qualquer forma, como parte da avaliação dos relaxantes musculares como nova tecnologia em saúde a ser incluída no SUS, talvez deva se considerar sua comparação com essas outras intervenções.

Nessa revisão, ciclobenzaprina demonstrou benefício no sono e na melhora geral dos pacientes com fibromialgia. Também demonstrou ser eficaz em casos de dor miofascial mandibular. Além disso, mostrou-se superior ao placebo no tratamento da dor lombar, porém com efeito modesto, mais presente nos quatro primeiros dias, o que sugere aconselhar seu uso por curtos períodos. ${ }^{26} \mathrm{O}$ profissional, ao prescrever esse medicamento, deve estar atento aos efeitos adversos citados em praticamente todas as pesquisas: sonolência e boca seca.

Quanto ao carisoprodol para dor lombar, apesar de não haver diferença significativa comparado com ciclobenzaprina, existem menos ensaios com esse fármaco. Além disso, carisoprodol possui potencial de provocar dependência física e psicológica. ${ }^{23}$ Dessa maneira, deveria ser reservado como última linha de terapia. ${ }^{37}$

Nos estudos encontrados pôde-se notar a constante dicotomia tizanidina versus blaclofeno que parecem demonstrar benefícios sobre o placebo quanto à melhora da espasticidade de modo geral. Porém, não se identificou diferenças entre estes fármacos. 
No Sistema Único de Saúde (SUS) nenhum relaxante muscular de uso oral descrito pela ATC faz parte da RENAME atualmente. ${ }^{4}$ Em parte, isso pode ocorrer devido à falta de conhecimento embasado em evidências científicas sobre a eficácia e a relação de custo-benefício destes medicamentos.

Assim, devido à popularidade e uso indiscriminado dos relaxantes musculares no tratamento de afecções frequentes na prática clínica da APS, é necessário que exista conhecimento, por parte dos profissionais, a respeito das evidências disponíveis sobre essa classe de medicamentos.

\section{Conclusão}

Os relaxantes musculares em geral comparados a placebo ou entre si apresentaram poucas evidências com estatística significante. Portanto, para uso ambulatorial, a seleção do medicamento deve ser baseada no perfil de efeitos adversos, preferência do paciente, potencial de abuso, potencial de interação com outros medicamentos, custo e outras características individuais dos fármacos. Não se pode afirmar que o uso isolado de relaxantes musculares ou mesmo associado a medicamentos com ação analgésica é benéfico a ponto de se considerar sua inclusão no Sistema Único de Saúde.

A maioria dos estudos incluídos nas revisões sistemáticas foi considerada de moderada qualidade, com problemas diversos como sigilo de alocação, cegamento, número de participantes pequeno, perda de seguimento e análise por protocolo. Em alguns cenários, não se pode descartar a ocorrência de viés de publicação, embora os autores tenham procurado minimizá-lo. Dessa forma, é necessário dar seguimento a essa pesquisa com busca por estudos primários com adequada qualidade metodológica e maior número de participantes, que avaliem a eficácia dos relaxantes musculares e considerem também seu uso como fármaco adjuvante.

\section{Referências}

1. Pereira LCM, L’Abbate GL. Miorrelaxantes no tratamento da dor. In: Alves Neto O, Costa CMC, Siqueira JTT, Teixeira MJ, orgs. Dor: Princípios e Prática. Porto Alegre: Artmed; 2009.

2. Duncan BB, Schmidt MI, Giuliani ERJ. Medicina Ambulatorial: Condutas de Atenção Primária Baseadas em Evidências. 4a ed. Porto Alegre: Artmed; 2013.

3. Associação da Indústria Farmacêutica de pesquisa [homepage na internet]. Guia 2017 Interfarma [acesso 27 Jan 2017 ]. Disponível em: https://www.interfarma.org.br/guia/guia-2017/dados-do-setor\#ranking-de-medicamentos

4. Brasil. Ministério da Saúde. Relação Nacional de Medicamentos Essenciais 2014 [Internet]. [acesso 17 Jun 2016]. Disponível em: http:// bvsms.saude.gov.br/bvs/publicacoes/relacao_nacional_medicamentos_essenciais_rename_2014.pdf

5. Rebolho MCT, Rocha LE, Teixeira LR, Casarotto RA. Prevalência de dor músculo esquelética e percepção de hábitos posturais entre estudantes do ensino fundamental. Rev Med [Internet]. Jun 2011 [acesso 18 Nov 2017];90(2):68-77. Disponível em: http://www.revistas. usp.br/revistadc/article/view/58887. DOI: http://dx.doi.org/10.11606/issn.1679-9836.v90i2p68-77

6. Brasil. Ministério da Saúde. Secretaria de Atenção à Saúde. Portaria Conjunta no. 29, de maio de 2017. Anexo Protocolo Clínico e Diretrizes Terapêuticas - Espasticidade. Diario Oficial da União 30 mai 2017; Seção 1 [Internet]. [acesso 24 Jan 2018]. Disponível em: http://conitec.gov.br/images/Protocolos/Protocolo_Uso/Portaria_SAS-SCTIE_2_PCDT_Espasticidade_29_05_2017.pdf

7. Witenko C, Moorman-Li R, Motycka C, Duane K, Hincapie-Castillo J, Leonard P, et al. Considerations for the appropriate use of skeletal muscle relaxants for the management of acute low back pain. P T. 2014;39(6):427-35.

8. WHO Collaborating Centre for Drug Statistics Methodology. Updates included in the ATC/DDD [Internet]. Nydalen: WHO/Norwegian Institute of Public Health. 2016 [atualizada em 2016 dez 19; acesso 22 Dez 2016]. Disponível em: https://www.whocc.no/atc_ddd_inde $\mathrm{x} /$ ?code=M03\&showdescription $=\mathrm{yes}$ 
9. Brasil. Ministério da Saúde. Agência Nacional de Vigilância Sanitária [Internet]. Consulta de Produto - Medicamentos [acesso 17 Jun 2016]. Disponível em: http://www7.anvisa.gov.br/datavisa/Consulta_Produto/consulta_medicamento.asp

10. Brasil. Ministério da Saúde. Secretaria de Ciência e Tecnologia e Insumos Estratégicos. Departamento de Ciência e Tecnologia. Diretrizes metodológicas: elaboração de pareceres técnico-científicos/Ministério da Saúde, Secretaria de Ciência e Tecnologia e Insumos Estratégicos, Departamento de Ciência e Tecnologia. $3^{a}$ ed., revisada e atualizada. Brasília: Ministério da Saúde; 2011.80 p.

11. Wallace JD. Summary of combined clinical analysis of controlled clinical trials with tizanidine. Neurology. 1994;44(11 Suppl 9):S60-8; discussion S68-9.

12. Manfredini D, Landi N, Tognini F, Orlando B, Bosco M. Muscle relaxants in the treatment of myofascial face pain. A literature review. Minerva Stomatol. 2004;53(6):305-13.

13. Lataste X, Emre M, Davis C, Groves L. Comparative profile of tizanidine in the management of spasticity. Neurology. 1994;44(11 Suppl 9):S53-9.

14. Groves L, Shellenberger MK, Davis CS. Tizanidine treatment of spasticity: a meta-analysis of controlled, double-blind, comparative studies with baclofen and diazepam. Adv Ther. 1998;15(4):241-51

15. Boothby LA, Doering PL, Hatton RC. Carisoprodol: a marginally effective skeletal muscle relaxant with serious abuse potential. Hosp Pharm. 2003;38(4):337-45. DOI: http://dx.doi.org/10.1177/001857870303800406

16. Gabriel SE, Bombardier C. Clinical trials in fibrositis: a critical review and future directions. J Rheumatol Suppl. 1989;19:177-9.

17. Tofferi JK, Jackson JL, O’Malley PG. Treatment of fibromyalgia with cyclobenzaprine: A meta-analysis. Arthritis Rheum. 2004;51(1):9-13. DOI: http://dx.doi.org/10.1002/art.20076

18. Roskell NS, Beard SM, Zhao Y, Le TK. A meta-analysis of pain response in the treatment of fibromyalgia. Pain Pract. 2011;11(6):516-27. DOI: http://dx.doi.org/10.1111/j.1533-2500.2010.00441.x

19. Richards BL, Whittle SL, Buchbinder R. Muscle relaxants for pain management in rheumatoid arthritis. Cochrane Database Syst Rev. 2012;1:CD008922.

20. Baldinger R, Katzberg HD, Weber M. Treatment for cramps in amyotrophic lateral sclerosis/motor neuron disease. Cochrane Database Syst Rev. 2012(4):CD004157. DOI: http://dx.doi.org/10.1002/14651858.CD004157.pub2

21. Peloso P, Gross A, Haines T, Trinh K, Goldsmith CH, Burnie S; Cervical Overview Group. Medicinal and injection therapies for mechanical neck disorders. Cochrane Database Syst Rev. 2007;(3):CD000319.

22. Leite FM, Atallah AN, El Dib R, Grossmann E, Januzzi E, Andriolo RB, et al. Cyclobenzaprine for the treatment of myofascial pain in adults. Cochrane Database Syst Rev. 2009(3):CD006830.

23. Chou R, Peterson K, Helfand M. Comparative efficacy and safety of skeletal muscle relaxants for spasticity and musculoskeletal conditions: a systematic review. J Pain Symptom Manage. 2004;28(2):140-75. DOI: http://dx.doi.org/10.1016/j.jpainsymman.2004.05.002

24. Vroomen PC, de Krom MC, Slofstra PD, Knottnerus JA. Conservative treatment of sciatica: a systematic review. J Spinal Disord. 2000;13(6):463-9. DOI: http://dx.doi.org/10.1097/00002517-200012000-00001

25. Luijsterburg PA, Verhagen A, Ostelo RW, van Os TA, Peul WC, Koes BW. Effectiveness of conservative treatments for the lumbosacral radicular syndrome: a systematic review. Eur Spine J. 2007;16(7):881-99. DOI: http://dx.doi.org/10.1007/s00586-007-0367-1

26. Browning R, Jackson JL, O’Malley PG. Cyclobenzaprine and back pain: a meta-analysis. Arch Intern Med. 2001;161(13):1613-20. DOI: http://dx.doi.org/10.1001/archinte.161.13.1613

27. Shakespeare DT, Boggild M, Young C. Anti-spasticity agents for multiple sclerosis. Cochrane Database Syst Rev. 2003(4):CD001332.

28. Paisley S, Beard S, Hunn A, Wight J. Clinical effectiveness of oral treatments for spasticity in multiple sclerosis: a systematic review. Mult Scler. 2002;8(4):319-29. DOI: http://dx.doi.org/10.1191/1352458502ms795rr

29. Beard S, Hunn A, Wight J. Treatments for spasticity and pain in multiple sclerosis: a systematic review. Health Technol Assess. 2003;7(40):iii, ix-x, 1-111.

30. Quality Standards Subcommittee of the American Academy of Neurology and the Practice Committee of the Child Neurology Society, Delgado MR, Hirtz D, Aisen M, Ashwal S, Fehlings DL, McLaughlin J, et al. Practice parameter: pharmacologic treatment of spasticity in children and adolescents with cerebral palsy (an evidence-based review): report of the Quality Standards Subcommittee of the American Academy of Neurology and the Practice Committee of the Child Neurology Society. Neurology. 2010;74(4):336-43. 
31. Navarrete-Opazo AA, Gonzalez W, Nahuelhual P. Effectiveness of Oral Baclofen in the Treatment of Spasticity in Children and Adolescents With Cerebral Palsy. Arch Phys Med Rehabil. 2016;97(4):604-18. DOI: http://dx.doi.org/10.1016/j.apmr.2015.08.417

32. Montané E, Vallano A, Laporte JR. Oral antispastic drugs in nonprogressive neurologic diseases: a systematic review. Neurology. 2004;63(8):1357-63. Epub 2004/10/27.

33. Taricco M, Pagliacci MC, Telaro E, Adone R. Pharmacological interventions for spasticity following spinal cord injury: results of a Cochrane systematic review. Eura Medicophys. 2006;42(1):5-15.

34. Marshall S, Teasell R, Bayona N, Lippert C, Chundamala J, Villamere J, et al. Motor impairment rehabilitation post acquired brain injury. Brain Inj. 2007;21(2):133-60. DOI: http://dx.doi.org/10.1080/02699050701201383

35. Lindsay C, Kouzouna A, Simcox C, Pandyan AD. Pharmacological interventions other than botulinum toxin for spasticity after stroke. Cochrane Database Syst Rev. 2016;10:CD010362. DOI: http://dx.doi.org/10.1002/14651858.CD010362.pub2

36. Olvey EL, Armstrong EP, Grizzle AJ. Contemporary pharmacologic treatments for spasticity of the upper limb after stroke: a systematic review. Clin Ther. 2010;32(14):2282-303. DOI: http://dx.doi.org/10.1016/j.clinthera.2011.01.005

37. See S, Ginzburg R. Choosing a skeletal muscle relaxant. Am Fam Physician. 2008;78(3):365-70.

Secretaria Municipal de Saúde de Florianópolis. Florianópolis, SC, Brasil. livia.helena.freitas@gmail.com (Autora correspondente); jardel_coli@yahoo.com.br 